\title{
Effects of Emotional Context during Encoding: An Advantage for Negative Context in Immediate Recognition and Positive Context in Delayed Recognition
}

\author{
Asako Toyama1 ${ }^{*}$, Maya Katsuhara ${ }^{2}$, Yoshio Sakurai ${ }^{3}$, Hideki Ohira1 \\ ${ }^{1}$ Department of Psychology, Graduate School of Environmental Studies, Nagoya University, Nagoya, Japan \\ ${ }^{2}$ RIKEN BSI-Toyota Collaboration Center (BTCC), RIKEN Brain Science Institute, Saitama, Japan \\ ${ }^{3}$ Department of Psychology, Graduate School of Letters, Kyoto University, Kyoto, Japan \\ Email: ${ }^{*}$ toyama.asako@b.mbox.nagoya-u.ac.jp
}

Received 29 April 2014; revised 28 May 2014; accepted 24 June 2014

Copyright (C) 2014 by authors and Scientific Research Publishing Inc.

This work is licensed under the Creative Commons Attribution International License (CC BY).

http://creativecommons.org/licenses/by/4.0/

(c) (i) Open Access

\begin{abstract}
This study examined the effect of emotional context on recognition memory for neutral targets over time. Participants $(\mathrm{N}=36)$ read sentences with negative, neutral, or positive valence in which emotionally neutral target words were embedded. Two groups of participants were asked to recognize targets after different retention intervals, immediate and 24-hour. Performance was better in the immediate recognition group than that in the delayed recognition group. The two groups showed opposite effects of emotional context: Target words in negative sentences were best remembered with immediate recognition but worst after a 24-hour delay. These results support the idea that emotional valence has different impacts on memory over time.
\end{abstract}

Keywords

Emotional Context, Recognition of Words, Retention Interval

\section{Introduction}

Events or objects that are essential or harmful are usually experienced with emotion, and memory for these emotional experiences provides important information when we encounter the same critical objects or events again.

\footnotetext{
${ }^{*}$ Corresponding author.
}

How to cite this paper: Toyama, A., Katsuhara, M., Sakurai, Y. and Ohira, H. (2014). Effects of Emotional Context during Encoding: An Advantage for Negative Context in Immediate Recognition and Positive Context in Delayed Recognition. Psychology, 5, 994-1000. http://dx.doi.org/10.4236/psych.2014.59111 
On the other hand, most objects and events that we experience daily are inherently emotionally neutral; however, if we encounter them in an emotional situation or context, they may take on the character of the simultaneously experienced emotion (De Houwer, Thomas, \& Baeyens, 2001). Many studies have focused on memory for emotional events or items, but relatively few have considered the effects of emotional contexts on neutral items embedded in these contexts. The aim of the present study was to examine the influence of contexts with different emotional valences on memory for inherently neutral items, based on numerous findings about the relationship between emotional stimuli and memory.

Studies of a variety of memory phenomena have investigated the components of emotional stimuli that enhance memory. For example, many studies have found that stimuli or events with high emotional intensity are more likely to be recognized than those with low emotional intensity, regardless of their emotional valence (Talarico, LaBar, \& Rubin, 2004). Other studies (e.g., Baumeister, Bratslavsky, Finkenauer, \& Vohs, 2001) have suggested that negative emotions have more impact than positive emotions, at least on short-term memory, because negative materials evoke more extensive conscious activity and receive deeper processing than positive materials, which in turn produces better memory. Thus there is a negativity bias in memory, except in some situations (e.g., when positive stimuli are related to the maintenance of self-esteem). However, in the case of autobiographical memory, which is relatively long-term, superior memory for positive everyday events, in comparison to negative or neutral events, has been widely reported (Berntsen, 1996; Wagenaar, 1986; Waldfogel, 1948).

The changing impact of different levels of emotional valence on memory over time suggests that there is a dynamical relationship between emotion and memory. The emotional intensity associated with an event becomes weaker over time, and episode recall tasks and diary studies have shown that this effect is larger for negative events than for positive events; this is known as the fading affect bias (FAB; see Walker \& Skowronski, 2009; Walker, Skowronski, \& Thompson, 2003, for reviews). This slower reduction of positivity than negativity may explain the memory advantages for positive events in comparison to negative events, especially in long-term autobiographical memory (Holmes, 1970). The difference in retention time is one of the important factors associated with the mixed influence of emotional valence on memory. Specifically, the FAB predicts that the superiority of memory performance for negative stimuli would rapidly diminish as the interval between encoding and recall increases. However, this hypothesis has not been examined systematically, primarily because of methodological limitations of previous studies. For example, in episode recall tasks, it is unclear how the events were initially encoded or how emotional factors were associated with the to-be-remembered objects. In diary studies, participants' self-editing of the to-be-remembered events may induce bias for reasons such as hesitation about exposing personal issues. To overcome these limitations of autobiographical memory, we developed a new paradigm using a modification and adaptation of the experimentally controlled procedure introduced by Banaji and Hardin (1994), which simulates autobiographical memory through the use of imaginary episodes.

In the original procedure, participants were instructed to write a description of an imagined, self-referential event using a neutral target word. This sentence had to reflect an experience of a given emotional valence. Using various target words, participants wrote negative, neutral, and positive sentences and were then given an unannounced free recall test for sentences or for target words. We modified this method by preparing a set of stimulus sentences in advance, instead of using stimuli created by participants. This modification was needed in our experiment to compare memory performance at different retention intervals using the same stimuli. We first created sentences that contained the same neutral word in different emotional contexts, and we conducted a preliminary study of the characteristics of these sentences. In the selection of the stimulus set, the concept of the "focus word" was considered. A focus word is the most important and critical component in the understanding of a sentence, and memory improves when a target word is also the focus word of the sentence (Osak, Nishizaki, Komori, \& Osaka, 2002). In our experiment, participants read each sentence and were given an incidental recognition test for the target words, either immediately after encoding (immediate recognition group), or 24 hours after encoding (delayed recognition group).

Using this paradigm, we investigated the influence of emotional valence on memory for neutral targets over time, and we examined the pattern predicted by the FAB. First, based on negativity bias or arousal, immediate recognition of a neutral target was predicted to be better in the negative condition, or in both negative and positive conditions, than in the neutral condition. Previous studies using similar methods have shown this pattern (Banaji \& Hardin, 1994; Phelps, LaBar, \& Spencer, 1997). We also expanded the investigation of the FAB. Specifically, we predicted that the difference in memory performance between the immediate and delayed recognition groups would be greater for stimuli with negative valence than for stimuli with positive or neutral valence. 


\section{Method}

\subsection{Preliminary Study}

To select target words, a preliminary study was conducted. Forty-seven neutral words were first selected from the list developed by Gotoh and Ohta (2001) in a study that surveyed the emotional valence and other attributes of words composed of two Japanese kanji characters. Each word was embedded in three first-person sentences with 37 to 42 Japanese hiragana characters, in which the emotional valence of context was manipulated at three levels: negative, neutral, and positive. These 141 (3 valences per a target word) sentences were randomly displayed to 19 volunteers ( 8 men, 11 women, Mean age 21.9 years, age range 19 to 24 years), who assessed the emotional valence of the sentences using a 5-point scale ranging between 1 (very negative) to 5 (very positive). They also rated their intensity of impressions on a 5-point ranging between 1 (not at all impressive) to 5 (very impressive), and determined which word was the focus word in each sentence. Based on the results of this preliminary study, we selected 39 target words (117 sentences) for the experiment and allocated them to three groups constructed of 13 targets words ${ }^{1}$. The characteristics of these stimuli are described in the section of 2.3 Instruments.

\subsection{Participants}

Undergraduate students $(\mathrm{N}=36)$ who were not involved in the preliminary study participated in the experiment. Individuals voluntarily consented to participate in an experiment with a personal computer. After all tasks were completed, participants were debriefed and told that the experiment was actually a study of memory. Half of the sample $(\mathrm{N}=18)$ was assigned to the immediate recognition group (6 men and 12 women, mean age $=21.6$, age range 20 - 24 years). The remainder of the sample $(\mathrm{N}=18)$ was assigned to the delayed recognition group (12 males and 6 females, mean age $=21.9$, age range $=21-24$ years).

\subsection{Instruments}

Thirty-nine neutral targets words (117 sentences) were classified into three groups, labeled A, B, and C. Stimulus lists were comprised of sentences with different emotional valences. For example, one stimulus list included 13 negative sentences from group A, 13 neutral sentences from group B, and 13 positive sentences from group C. Six lists were created for the experiment and each participant completed one of the six lists. Table 1 includes the attributes of sentences used in the experiment. Additional 39 neutral words were selected from the list by Gotoh and Ohta (2001) as new words in the recognition test. The stimuli were displayed on the screen at 16-point fontsize (black letters with no line breaks on a white background), at a viewing distance of $45 \mathrm{~cm}$, each item subtended a horizontal visual angle of $31^{\circ}$ and a vertical angle of $15^{\circ}$. Examples of the stimulus sentences transformed to English are shown below.

Target word: script

- Negative sentence: I spilled sauce on my drama script, which is precious to me, but I had to keep using it.

- Neutral sentence: When I went to the gymnasium on an errand, I found a script on the stage left there by a member of the drama club.

- Positive sentence: As only I remembered the script perfectly, I gained the director's confidence.

\subsection{Procedure}

The experiment was composed of three tasks: a rating task, a filler task, and a recognition task. Participants in the immediate recognition group completed the recognition task immediately after the filler task. Participants in the delayed recognition group completed the recognition task 24-hour after the filler task. Participants were not informed about the recognition task that was to follow.

In the rating task, 39 sentences were randomly presented. In each trial, a sentence was displayed on the screen for 14 seconds. Participants were instructed to read the sentence aloud once and to imagine the context vividly in

\footnotetext{
${ }^{1}$ In this process, we considered the following criteria. 1) Rating of valences for neutral sentences should be approximately 3.0. 2) Rating of valences for negative and positive sentences should be almost the same distances but opposite directions from 3.0. 3) Ratings of the impression of a sentence in each valence condition should be about the same. 4) The degree to which target words coincide with the focus words of sentences should be about the same. 5) Ratings of emotional valence, imagination, and ease of learning target words should be approximately the same. The criterion 5) was checked based on a study by Gotoh and Ohta (2001).
} 
Table 1. Sentence attributes based on results of the preliminary study (emotional valence, impression, and focus).

\begin{tabular}{ccccc}
\hline Sentence valence & Group & Valence & Impression & Focus \\
\hline \multirow{2}{*}{ Negative } & Group A & $1.8(0.27)$ & $3.7(0.28)$ & $0.51(0.31)$ \\
& Group B & $1.8(0.20)$ & $3.5(0.27)$ & $0.47(0.24)$ \\
& Group C & $1.8(0.29)$ & $3.8(0.32)$ & $0.58(0.32)$ \\
\multirow{2}{*}{ Neutral } & Group A & $3.0(0.19)$ & $2.9(0.23)$ & $0.56(0.23)$ \\
& Group B & $3.0(0.21)$ & $2.8(0.26)$ & $0.60(0.24)$ \\
& Group C & $3.1(0.16)$ & $2.9(0.25)$ & $0.54(0.24)$ \\
Positive & Group A & $4.2(0.26)$ & $3.5(0.38)$ & $0.56(0.29)$ \\
& Group B & $4.1(0.22)$ & $3.6(0.19)$ & $0.52(0.26)$ \\
\hline
\end{tabular}

Note: Values in parentheses represent standard deviations. "Focus” refers to the proportion that a target word was regarded as the central word in the sentence.

order to amplify emotional feelings evoked by the sentence. Next, participants rated the ease of imaging (if it was easy for them to imagine the scene with $1=$ not at all and $5=$ very vividly) and the emotional valence (how they felt about this sentence using a 5 -point scale ranging with $1=$ very negative and $5=$ very positive). This process was repeated for all 39 sentences. At the end of each sentence set, a black fixation-cross appeared on the screen for 1 second.

Immediately after the rating task, participants performed the filler task, designed to reduce the recency effect. The filler task consisted of simple mathematical calculations. Following the filler task, two groups performed a recognition task at a certain delay time. For the recognition task, the original 39 neutral target words presented earlier (i.e., "old" words) were combined with 39 other neutral words that had not been previously presented (i.e., "new" words). Old and new words were randomly presented on the center of the computer screen. Participants were asked to categorize all 78 words as either "old" or "new" by pressing a corresponding key.

After the recognition task, participants were asked whether they had been able to anticipate a later memory test during the rating task. All 36 participants answered that they could not.

\section{Results}

\subsection{Sentence Ratings}

\subsubsection{Ease of Imagining}

An analysis of variance (ANOVA) on scores of easiness to imagine was conducted, with Delay (immediate recognition and delayed recognition) as a between-subjects factor and Valence of sentence (negative, neutral, and positive) as a within-subjects factor. There was no significant main effect for Valence $(\mathrm{F}(2,68)=0.01$, MSE $=$ $0.30)$, or Delay $(F(1,34)=0.00$, MSE $=0.71)$, and no significant interaction $(F(2,68)=0.21$, MSE $=0.30)$. The mean values are shown in Table 2.

\subsubsection{Emotional Valence}

An ANOVA on scores of emotional valence was also conducted, with Delay and Valence of sentence. It revealed a significant main effect of Valence $(F(2,68)=1522.63$, MSE $=0.10, p<0.01)$. Scores were highest in the positive condition followed by the neutral and negative conditions with a significant difference, $p<0.01$ for all comparisons. There was neither a main effect of Delay $(F(1,34)=1.35$, MSE $=0.09)$, nor an interaction $(F(2$, $68)=0.42$, MSE $=0.08$ ). This result confirms that the emotional valences of sentences were appropriately manipulated. The mean values are shown in Table 2.

\subsection{Recognition Memory}

Hit rates were calculated for three valence conditions and an ANOVA on hit rate was conducted, with Delay and Valence of sentence. Results revealed a significant main effect of Delay $(\mathrm{F}(1,34)=18.46$, MSE $=0.06, p<$ 
Table 2. Means of sentence ratings.

\begin{tabular}{cccc}
\hline & Sentence valence & Immediate recognition group & Delayed recognition group \\
\hline \multirow{2}{*}{ Ease of imagining } & Negative & $3.78(0.60)$ & $3.77(0.49)$ \\
& Neutral & $3.85(0.66)$ & $3.67(0.53)$ \\
& Positive & $3.76(0.65)$ & $3.75(0.61)$ \\
Emotional valence & Negative & $1.52(0.26)$ & $1.65(0.31)$ \\
& Neutral & $3.30(0.37)$ & $3.30(0.25)$ \\
\hline
\end{tabular}

Note: Values in parentheses represent standard deviations.

0.01 ), suggesting that targets were better recognized in the immediate recognition group than in the delayed recognition group. There was no significant main effect of Valence $(F(2,68)=0.32$, MSE $=0.01)$. A significant interaction between Delay and Valence was found $(\mathrm{F}(2,68)=10.98$, MSE $=0.01, p<0.01)$. Post-hoc analysis using Tukey's WSD revealed that targets in negative sentences were recognized better than targets in neutral and positive sentences in the immediate condition $(p<0.05)$, but worse in the delayed condition $(p<0.05)$. No other pairs differed significantly. Figure 1 shows the differentiated reduction of hit rates and discriminability between two groups among the three valence conditions, with the largest difference for negative targets followed by neutral and positive targets.

\section{Discussion}

A variety of reports have provided inconsistent results about enhancement of memory in the context of different emotional valences. The present study examined retention time as a possible reason for this discrepancy. Recognition memory was found to be affected by the level of emotional valence that was experienced during the encoding phase, but the pattern of effects differed depending on when the recognition was required. That is, in immediate recognition, target words in negative sentences were recognized better than those in positive or neutral sentences. Memory performance was worse for delayed recognition than for immediate recognition for all valence levels. Contrary to the results in the immediate recognition task, target words in positive and neutral sentences were recognized better after a delay than those in negative sentences.

One explanation for enhanced memory in the negative condition for the immediate recognition group may come from response bias. This bias is more often seen with negative than with neutral or positive words and increases subjective reports of "remembering" (Dougal \& Rotello, 2007). Because emotional information was added to the neutral targets, immediate memory performance may have shown the typical pattern caused by this bias. Good memory for negative items may also be explained in terms of their adaptive function. That is, negative stimuli generally contain more urgent or survival-relevant information (Pratto \& John, 1991), which we should pay more attention to. However, such bias or stimulus-based features cannot explain the reversed memory performance demonstrated in the present study.

The reversed memory pattern seen here may be explained by the fading affect bias. In the FAB phenomenon, the intensity of negative emotion fades faster than that of positive emotion (Holmes, 1970; Thompson, 1985; Walker, Vogl, \& Thompson, 1997). Given this bias, the reduced impact of negative intensity may have caused the largest reduction of recognition performance in the negative condition within a 24-hour delay period and also led to worse recognition of negative than positive conditions at the 24-hour delay. The FAB has been demonstrated exclusively in association with relatively long retention periods, from a few weeks to a few months (Walker et al., 1997). Although it has recently been reported that the FAB effect also occurs within one day (Gibbons, Lee, \& Walker, 2011), until now, no information has been available about corresponding delays for memory performance. Furthermore, although the FAB has previously been reported only for emotional events, the present study used neutral stimuli embedded in an emotional context at encoding and revealed results that correspond to the predictions of the FAB.

The reason for the FAB effect is still under investigation (but see Ritchie, Skowronski, Hartnett, Wells, \& Walker, 2009). Walker et al. (2003) cited the mobilization-minimization hypothesis developed by Taylor (1991), which distinguishes negative from positive and neutral events and is applicable to our pattern of results. 


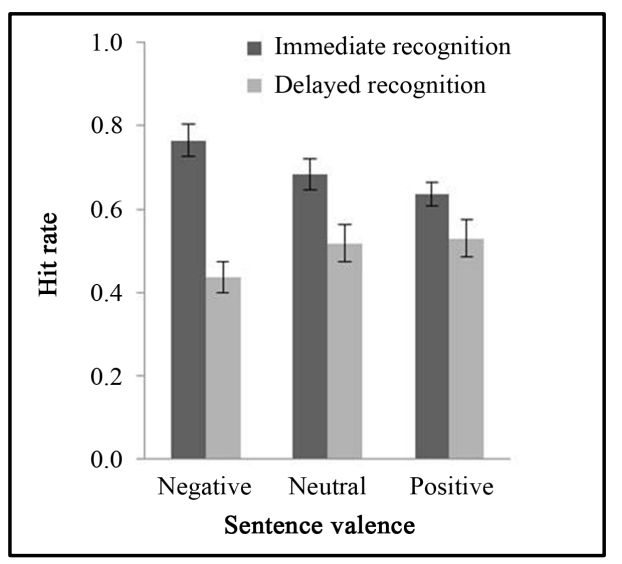

Figure 1. Means of hit rates for targets in conditions of sentence valence; negative, neutral and positive. Error bars show standard errors.

According to this hypothesis, when a negative event occurs, people are strongly mobilized to cope with the immediate consequence of that event, which may account for the immediate enhanced memory for target words with emotionally negative valences that was found in our study. On the other hand, to maintain homeostasis, people activate opponent processes with the goal of minimizing the impact of the event. This explanation is compatible with our results and also has been supported by previous findings of long-term memory tasks requiring diary records or recall of childhood events (Wagenaar, 1986; Waldfogel, 1948).

The comparison of immediate and 24-hour delay has also drawn attention in sleep studies. Emotional images or sentences have often been found to facilitate memory both immediately and after sleep. However, to the best of our knowledge, the pattern seen in the current data have not been previously reported. For example, Lewis, Cairney, Manning, and Critchley (2011) reported no difference between the influences of negative and neutral context on memory when neutral images were used as retrieval cues to remember context. Another study reported better recollection of words included in negative sentences than in neutral sentences when recall occurred both immediately and after sleep (Wagner, Degirmenci, Drosopoulos, Perras, \& Born, 2005). However, the methodological differences between these studies and the present research make a direct comparison difficult. Lewis et al. (2011) examined memory for context by using neutral targets as cues, and Wagner et al. (2005) examined memory for any words in the sentences, not only neutral words. Further experiments will clarify which aspects of memory are characterized by the reversed pattern.

Of course, there are some limitations to our interpretation of the present pattern of results. First, emotionally qualified items were not always remembered better than purely neutral items. Contrary to our results, previous studies using a similar method have found that emotional items were often associated with better memory performance regardless of their emotional valences (i.e., positive or negative) relative to neutral items (Banaji \& Hardin, 1994; Phelps et al., 1997). It may have been more difficult to evoke positive arousal than negative arousal because the stimuli in our experiment were prepared sentences, although the valence scores of negative and positive conditions were equal distances from the neutral condition. Further, recognition memory was recorded at only two time points. As Gibbons et al. (2011) indicated, FAB could occur within a single test day. Thus future studies should add temporally separated observation points in order to capture continuous changes in memory to validate and refine the pattern demonstrated in the current experiment.

\section{Conclusion}

The current task yielded a unique reversed pattern of memory performance at immediate and 24-hour delays. Although this reversal has rarely been seen in previous studies, the memory patterns at the two respective test points coincided with previous findings. We revealed this valence-specific recognition pattern for neutral targets using well-controlled episodic stimulus sets. Participants' ratings of emotional valence for each sentence confirmed the validity of the emotional categories used in the study. A form of cognitive bias may have contributed to these results. Together, these findings and method offer a new perspective on memory for initially neutral targets as colored by emotional information. 


\section{Acknowledgements}

I wish to express my deepest appreciation to Dr. Hiroshi Ashida for providing helpful comments and suggestions.

\section{References}

Banaji, M. R., \& Hardin, C. (1994). Affect and Memory in Retrospective Reports. In N. Schwarz, \& S. Sudman (Eds.), Autobiographical Memory and the Validity of Retrospective Reports (pp. 71-86). New York: Springer-Verlag. http://dx.doi.org/10.1007/978-1-4612-2624-6 6

Baumeister, R. F., Bratslavsky, E., Finkenauer, C., \& Vohs, K. D. (2001). Bad Is Stronger than Good. Review of General Psychology, 5, 323-370. http://dx.doi.org/10.1037//1089-2680.5.4.323

Berntsen, D. (1996). Involuntary Autobiographical Memories. Applied Cognitive Psychology, 10, 435-454. http://dx.doi.org/10.1002/(SICI)1099-0720(199610)10:5<435::AID-ACP408>3.0.CO;2-L

De Houwer, J., Thomas, S., \& Baeyens, F. (2001). Associative Learning of Likes and Dislikes: A Review of 25 Years of Research on Human Evaluative Conditioning. Psychological Bulletin, 127, 853-869. http://dx.doi.org/10.1037/0033-2909.127.6.853

Dougal, S., \& Rotello, C. M. (2007). “Remembering” Emotional Words Is Based on Response Bias, Not Recollection. Psychonomic Bulletin \& Review, 14, 423-429. http://dx.doi.org/10.3758/BF03194083

Gibbons, J. A., Lee, S. A., \& Walker, W. R. (2011). The Fading Affect Bias Begins within 12 Hours and Persists for 3 Months. Applied Cognitive Psychology, 25, 663-672. http://dx.doi.org/10.1002/acp.1738

Gotoh, F., \& Ohta, N. (2001). Affective Valence of Two-Compound Kanji Words. Tsukuba Psychology Research, $23,45-52$.

Holmes, D. S. (1970). Differential Change in Affective Intensity and the Forgetting of Unpleasant Personal Experiences. Journal of Personality and Social Psychology, 15, 234-239. http://dx.doi.org/10.1037/h0029394

Lewis, P. A., Cairney, S., Manning, L., \& Critchley, H. D. (2011). The Impact of Overnight Consolidation upon Memory for Emotional and Neutral Encoding Contexts. Neuropsychologia, 49, 2619-2629. http://dx.doi.org/10.1016/j.neuropsychologia.2011.05.009

Osak, M., Nishizaki, Y., Komori, M., \& Osaka, N. (2002). Effect of Focus on Verbal Working Memory: Critical Role of the Focus Word in Reading. Memory \& Cognition, 30, 562-571. http://dx.doi.org/10.3758/BF03194957

Phelps, E. A., LaBar, K. S., \& Spencer, D. D. (1997). Memory for Emotional Words Following Unilateral Temporal Lobectomy. Brain and Cognition, 35, 85-109. http://dx.doi.org/10.1006/brcg.1997.0929

Pratto, F., \& John, O. P. (1991). Automatic Vigilance: The Attention-Grabbing Power of Negative Social Information. Journal of Personality and Social Psychology, 61, 380-391. http://dx.doi.org/10.1037/0022-3514.61.3.380

Ritchie, T., Skowronski, J. J., Hartnett, J., Wells, B., \& Walker, W. R. (2009). The Fading Affect Bias in the Context of Emotion Activation Level, Mood, and Personal Theories of Emotion Change. Memory, 17, 428-444. http://dx.doi.org/10.1080/09658210902791665

Talarico, J. M., LaBar, K. S., \& Rubin, D. C. (2004). Emotional Intensity Predicts Autobiographical Memory Experience. Memory \& Cognition, 32, 1118-1132. http://dx.doi.org/10.3758/BF03196886

Taylor, S. E. (1991). Asymmetrical Effects of Positive and Negative Events: The Mobilization-Minimization Hypothesis. Psychological Bulletin, 110, 67-85. http://dx.doi.org/10.1037//0033-2909.110.1.67

Thompson, C. P. (1985). Memory for Unique Personal Events: Effects of Pleasantness. Motivation and Emotion, 9, $277-289$. http://dx.doi.org/10.1007/BF00991832

Wagenaar, W. A. (1986). My Memory: A Study of Autobiographical Memory over Six Years. Cognitive Psychology, 18, 225-252. http://dx.doi.org/10.1016/0010-0285(86)90013-7

Wagner, U., Degirmenci, M., Drosopoulos, S., Perras, B., \& Born, J. (2005). Effects of Cortisol Suppression on SleepAssociated Consolidation of Neutral and Emotional Memory. Biological Psychiatry, 58, 885-893. http://dx.doi.org/10.1016/j.biopsych.2005.05.008

Waldfogel, S. (1948). The Frequency and Affective Character of Childhood Memories. Psychological Monographs: General and Applied, 62, i-39. http://dx.doi.org/10.1037/h0093581

Walker, W. R., \& Skowronski, J. J. (2009). The Fading Affect Bias: But What the Hell Is It for? Applied Cognitive Psychology, 23, 1122-1136. http://dx.doi.org/10.1002/acp.1614

Walker, W. R., Skowronski, J. J., \& Thompson, C. P. (2003). Life Is Pleasant-And Memory Helps to Keep It That Way! Review of General Psychology, 7, 203-210. http://dx.doi.org/10.1037/1089-2680.7.2.203

Walker, W. R., Vogl, R. J., \& Thompson, C. P. (1997). Autobiographical Memory: Unpleasantness Fades Faster than Pleasantness over Time. Applied Cognitive Psychology, 11, 399-413.

http://dx.doi.org/10.1002/(SICI)1099-0720(199710)11:5<399::AID-ACP462>3.0.CO;2-E 
Scientific Research Publishing (SCIRP) is one of the largest Open Access journal publishers. It is currently publishing more than 200 open access, online, peer-reviewed journals covering a wide range of academic disciplines. SCIRP serves the worldwide academic communities and contributes to the progress and application of science with its publication.

Other selected journals from SCIRP are listed as below. Submit your manuscript to us via either submit@scirp.org or Online Submission Portal.
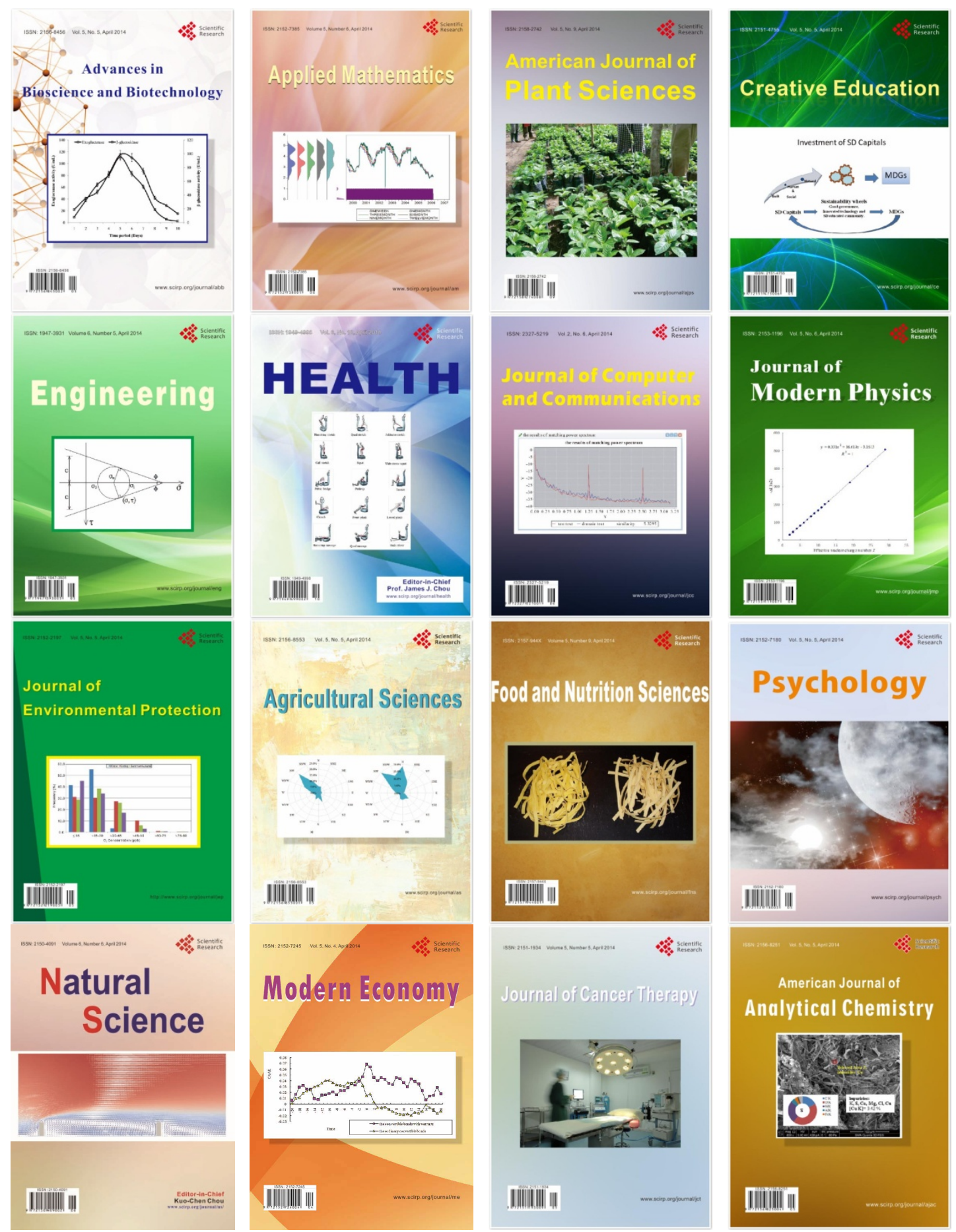